\title{
A CASE REPORT: CLINICAL INSIGHT ON RETAINED WOODEN FOREIGN BODY IN THE FOOT
}

KEY WORDS: Retained wooden foreign body, foot, subtalar, foot injury, foreign body.

\section{Dr.M.Christan}

\section{Dr.S.Daniel} Sundar Singh*

Tabassum

Fathima

\section{Prukruthi R}

Clinical Research Department,gleneagles Global Hospital,chennai,tamil Nadu.

Assistant Professor, Department of Pharmacy practice,C.L.Baid Metha College of Pharmacy Chennai,Tamil Nadu. *Corresponding Author

Department of Pharmacy practice,C.L.Baid Metha College of Pharmacy Chennai,Tamil Nadu.

Department of Pharmacy practice,C.L.Baid Metha College of Pharmacy Chennai,Tamil Nadu.

The foot is the second commonest location for foreign bodies. The foremost common foreign bodies are needles, metal, glass, wood, and plastic. Though bimetal foreign bodies area unit promptly seen on plain film radiographs, radiolucent bodies like wood area unit pictured poorly, if at all.Though plain radiography is thought to be ineffective for demonstrating radiolucent foreign bodies, it's usually the primary imaging modality used. Herein, we present the case of a 42 years old man who had presented to the clinic with history of pain and swelling in his right foot. On examination, his vitals, heart sounds and breathing during auscultation were found to be normal. This particular individual had no other significant chronic illness. A brief history obtained from the patient revealed that penetrated wooden foreign bodies in his hind sole region. In view of his present complaints, he was successfully managed with antibiotics and pain relieving medications. Our patient comes under the small percentage of cases that had a missed diagnosis as the expulsion of the wooden particles occurred 3 months after the initial visit to the clinic. This case is being presented to enlighten understanding on clinical picture of retained wooden foreign bodies in foot.

\section{INTRODUCTION:}

The foot is one of the most common parts of the body exposed to the external environment. The practice of walking barefoot increases the incidence of injuries on the feet due to foreign bodies. Needles, metal, glass, wood, plastic, mud and stones are the most common foreign bodies encountered.

Foreign substances in any part of the body cause a cascade of inflammatory reactions. Patients can experience local and systemic symptoms such as pain, swelling, and fever.They can also be a source of infections, which, depending on the severity, might require further treatment and hospitalisation.

Imaging is the main method of diagnosis involving suspected injuries due to retained foreign bodies as it provides their exact location. Radiography, CT, MRI and sonography are the mainstay diagnostic techniques with $\mathrm{X}$-ray imaging being the first modality. Conventional radiography is useful to detect radiopaque and metal substances.

Despite advances in imaging techniques, the detection of retained wooden foreign bodies remains a challenging task. ${ }^{[1]}$ The radiolucent nature of wood makes it imperceptible on radiographs. Hence, the imaging of wooden foreign bodies goes undetected in many cases.

Wooden fragments account for the largest proportion of retained foreign bodies after trauma to the human body. ${ }^{[2]}$ Such bodies can be detected months or years after the initial injury and is often accompanied by painful, swollen soft-tissue mass that usually suggests a malignancy or infection. Detecting wooden foreign bodies is essential as its porous consistency andorganic nature can be an unrecognised nidus for infection, causing cellulitis, abscess or fistula formation. ${ }^{[3]}$

Wooden foreign bodies can now be detected through ultrasound, CT or MRI techniques. Ultrasound has been found to have asensitivity of 50 to 90 percent and a specificity of 70 to 97 percent for gravel, metal, cactus spines, wood, and plastic. ${ }^{[4]}$ Additionally, due to easy availability, cost effectiveness, and lack of exposure to ionising radiation, ultrasound techniques are more commonly used to detect foreign bodies. ${ }^{[5]}$

The treatment for suspected wooden foreign body injuries is subjective. If the foreign body can be visualised, a surgical procedure to remove it is performed.Removal is easier if wounds are examined within 24 hours asentry wounds are visible and open. ${ }^{[6]}$ When the foreign body is invisible, detailed medical histories to rule out other causes of symptoms is to be done. Diagnosis must be confirmed through imaging techniques and wound exploration. Surgical procedures can then be performed to retrieve it. Management of accompanying symptoms is supportive. Patients experiencing pain can be prescribed analgesics. Antibiotic prophylaxis is generally not required. However, in case of suspected infections, empirical antibiotic therapy should be initiated and switched appropriately after confirming the presence and type of causative organism. Tetanus prophylaxis is necessary if there is no knowledge or documentation of tetanus immunization within 10 years. ${ }^{[6]}$

We present a case report that follows the course of events after a patient had two pieces of wooden foreign bodies penetrated his right foot.

\section{CASE REPORT:}

A 42 year old male patient presented to the local clinic with a history of pain and swelling on his right foot. On examination, his vitals, heart sounds and breathing were found to be normal. He has no known history of any other chronic diseases such as diabetes mellitus, hypertension, and kidney disease. This individual is otherwise healthy and well.

A brief history obtained from the patient revealed that, while plowing land, he felt something had gotten stuck in his right foot, particularly in the hind sole(figure:A). At the time, the patient wasunaware of the foreign body inside the foot. After this event, he was unable to perform his routine work due to his inabilityto walk as a result of severe pain and swelling. (figure:A)showed a healed penetrating site. Unfortunately, we 
were unable to get the image at the time of injury.

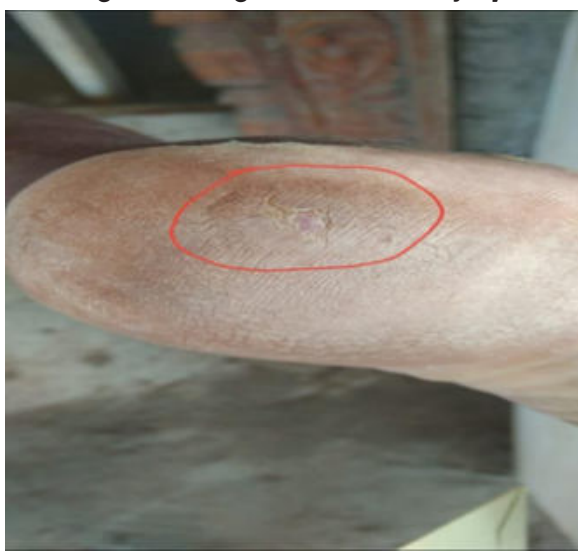

\section{Figure A: Image view of Hindsole}

On the 2nd day, the patient went to a local clinic. He underwent foot examination where the physician made a small incision to identify any embedded foreign body on the site of injury.No foreign bodies were found.

The patient was given Inj.TT and T. Diclofenac sodium with Paracetamol combination to relieve pain. This particular individual's X-Ray of the right foot revealed that no foreign bodies were identified(figure:B).He was prescribed oral Ofloxacin $200 \mathrm{mg}$ BD for 4 days. After the 7 th day, the patient failed to go for further review.

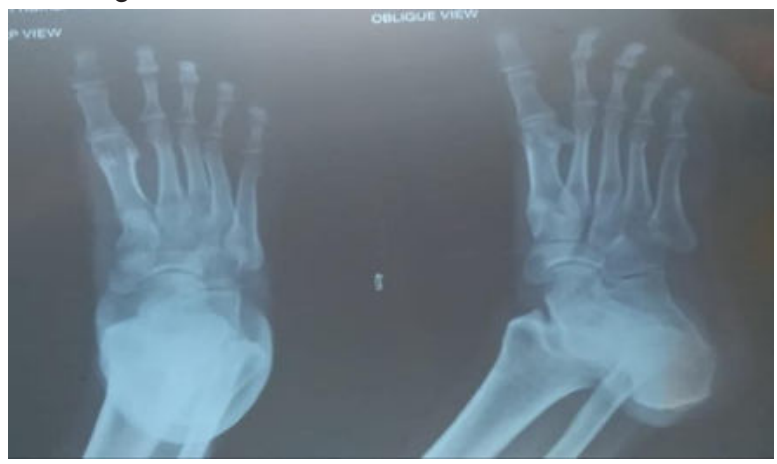

Figure B: X-ray of Right foot

Later, the patient developed a small pustule over the sub-talar joint region of the right foot. The location of this pustule was directly opposite of the already existing wound on the hindfoot region. With time, the hind foot injury had healed.(figure: A shows healed hind foot injury).

On the 90th day, the patient had noticed 2 tiny pieces of wooden particles expelled out automatically from the newly formed pustules located on the opposite side of the injured site beside the sub-talar joint region(figure:C).

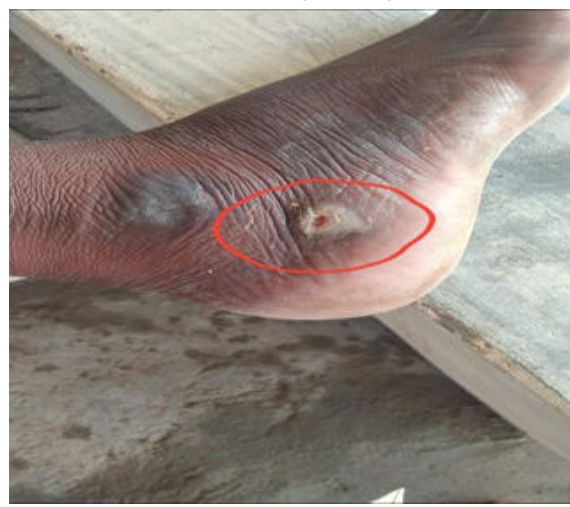

Figure C: Image view of Sub-talar joint region
The expelled wooden particles' size was estimated to be $1 \mathrm{~cm}$ each(figure:D).
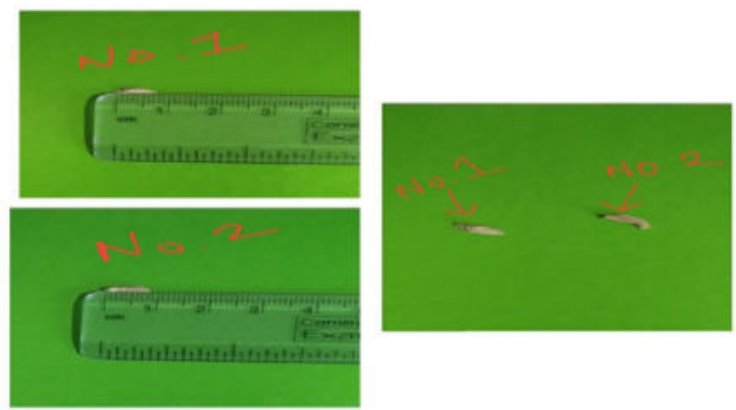

Figure D: Size ofWooden particles

The patient returned to the local clinic with the 2 tiny wooden pieces that were retained in his right foot for 3 months. This individual is now healthy and able to perform his daily routine.

\section{DISCUSSION:}

Penetrating foreign body injuries can be caused by various materials like metals, glass, wood, or plastic objects. The extremities are the most common places of penetrative injuries. Hiremathet al., through their prospective study found that most of the foreign body reactions were noted in the lower limbs. ${ }^{[5]}$ Another study by Polatet al., found that the feet and hands were the most affected parts of the body. ${ }^{[7]}$ Similarly, through their literature review, Dhillonet al., found that the metatarsal bone was the most common site of the feet for pseudo tumor injuries due to wooden foreign bodies. ${ }^{[8]}$

A thorough physical examination is required as foreign bodies are frequently missed during initial evaluations. A retrospective study conducted by Anderson et al., identified that nearly $38 \%$ of the patients with foreign bodies were missed on initial wound inspection. ${ }^{[2]}$ Levine et al., found that $25 \%$ of the patients with soft tissue foreign bodies presented within weeks, months, and even years later after the injury. ${ }^{[9]}$ Our patient comes under the small percentage of cases who had a missed diagnosis as the expulsion of the wooden particles occurred 3 months after the initial visit to the clinic.Our present case warrants an early and accurate diagnosis as wooden particles are conducive for microbial growth and can produce a granulomatous reaction.

Radiologic visualization is required unless the remnant is palpable or discernible from the outside.$^{[7]} \mathrm{X}$-rays are useful but studies suggest that it could detect only $15 \%$ of the wood particles that are embedded. ${ }^{[2]}$ The same had occurred with our patient where plain radiologic examination, which was the only modality used, could not detect any wooden particles.

The disadvantage of radiography can be overcome by switching over to ultrasonography, especially for penetrant injuries by wooden particles. Rockettet al., reported in their study that ultrasound was 100 percent sensitive in detecting wooden bodies in the soft tissues of the foot. ${ }^{[10]}$ Through ultrasound, wooden particles are visualized as hyperechoic regions and the adjacent soft tissues appear hypoechoic due to inflammation, edema and granuloma formation. ${ }^{[11]}$ Detection then becomes easier and surgical extraction can be performed. CT and MRI are also excellent techniques but have the disadvantages of higher cost, limited availability and exposure to radiation.

Pain is a common manifestation due to inflammation of the injured tissues. This is supported by Peterson et al., whose study showed that pain and swelling were the most common symptoms their patients had experienced. ${ }^{[1]}$ In our case, the patient had severe pain with swelling for which analgesics were prescribed. Studies show that some cases remain 
asymptomatic for a longer period of time with one report by Gulatiet al., showing symptomatic presentation 8 years after injury. ${ }^{[12]}$

Our patient was also prescribed with a fluoroquinolone antibiotic as prophylaxis. Since the wooden particles were expelled out automatically, there was no surgical intervention done to the patient.In the last three decades, various reports have been published regarding the diagnosis and treatment of wooden foreign bodies. Many cases showed that there was a period of delay before the diagnosis was confirmed. This accentuates the rarity of the problem and the need for awareness among healthcare professionals and the public. Keeping this in mind, further research into advanced imaging techniques is requiredthat can accurately describe the characteristics of wooden foreign bodiesand locate them timely to prevent further complications, decrease the financial burden of treatment to the patients and improve overall health care.

\section{CONCLUSION:}

Retained foreign bodies are common causes of injury to the feet. The diagnosis of wooden foreign bodies through imaging is tricky due to its innate nature and hence requires further research. The investigation of a suitable diagnostic technique that is cost effective, easily available and accurate is essential to prevent serious complications and improve overall health of such patients.

\section{Conflict Of Interest:}

NIL

\section{Acknowledgement:}

The author would like to thank the patient who has participated in this Report.

\section{REFERENCES:}

1. Peterson JJ, Bancroft LW, Kransdorf MJ. Wooden foreign bodies: imaging appearance. AJR Am J Roentgenol 2002;178:557-62.

2. Anderson MA, Newmeyer WL 3rd, Kilgore ES Jr. Diagnosis and treatment of retained foreign bodies in the hand. AmJ Surg 1982;144:63-7.

3. Peterson JJ, Bancroft LW, Kransdorf MJ. Wooden foreign bodies: imaging appearance. AJR Am J Roentgenol.2002 Mar;178(3):557-62.

4. Capellan O, Hollander JE. Management of lacerations in the emergency department.Emerg Med Clin North Am. 2003;21:205-31.

5. Hiremath R, Reddy H, Ibrahim J, Haritha $\mathrm{CH}$, Shah RS. Soft Tissue Foreign Body: Utility of High Resolution Ultrasonography. J Clin Diagn Res. 2017 Jul;11(7):TC14-TC16.

6. Halaas GW. Management of foreign bodies in the skin. Am Fam Physician. 2007 Sep 1;76(5):683-8.

7. Polat B, Atici Y, Gürpinar T, Polat AE, Karagüven D, Benli IT. Diagnosis and Treatment of Retained Wooden Foreign Bodies in the Extremities Using Ultrasound. ActaOrtop Bras. 2018 May-Jun;26(3):198-200.

8. Dhillon MS, Prasanna HM, Goni V, Nagi ON.Wooden splinter-induced pseudo tumour of the metatarsal. Foot Ankle Surg. 2000;6(1):45-8.

9. Levine MR, Gorman SM, Young CF, Courtney DM. Clinical characteristics and management of wound foreign bodies in the ED. Am J Emerg Med. 2008 Oct. 26(8):918-22.

10. Rockett MS, Gentile SC, Gudas CJ, Brage ME, Zygmunt KH. The use of ultrasonography for the detection of retained wooden foreign bodies in the foot.J Foot Ankle Surg. 1995 Sep-Oct;34(5):478-84; discussion 510-1.

11. Shiels WE, Babcock DS, Wilson JL, Burch RA. Localization and guided removal of soft- tissue foreign bodies with sonography. AJR Am J Roentgenol. 1990 Dec;155(6):1277-81.

12. Gulati D, Agarwal A. Wooden foreign body in the forearm - presentation aftereight years. UlusTravmaAcilCerrahiDerg. 2010;16(4):373-5. 